Metacognitive Awareness in the Sound-Induced Flash Illusion

Callie E. Mims, Brian Odegaard

University of Florida

\begin{abstract}
Author Note
Callie E. Mims does not have an ORCID.

Brian Odegaard: https://orcid.org/0000-0002-5459-1884

Brian Odegaard is currently at the University of Florida in Gainesville, FL.

We have no conflicts of interest to disclose.

Correspondence concerning this article should be addressed to: Brian Odegaard, University of Florida Psychology Department, 945 Center Dr, Gainesville, FL 32603, Email: bodegaard@ufl.edu
\end{abstract}




\begin{abstract}
In the sound-induced flash illusion, discrepant numbers of stimuli in the visual and auditory modalities are integrated to create illusory visual percepts. While this phenomenon has been extensively studied over the last two decades, relatively little is known about the role of metacognition in this illusion. Specifically, when incongruent audiovisual signals yield the same behavioral reports as conditions with congruent audiovisual signals, is confidence in the perceptual judgment the same across conditions? To probe this question, we conducted an experiment where 22 observers viewed from 1-4 flashes and 1-4 beeps on each trial, and reported three things: (1) the number of perceived flashes, (2) confidence in the judgment about the number of flashes, and (3) confidence in whether the number of beeps and flashes were the same or different. In our exploratory analyses, we paired conditions of incongruent and congruent audiovisual signals which produced the same report about the number of flashes. Results showed that in several condition pairs, confidence in the perceived number of flashes was higher for congruent audiovisual signals than incongruent audiovisual signals. For confidence judgments about whether the number of auditory and visual signals presented were the same or different, confidence ratings were similar for all condition pairs but one. These findings provide preliminary support for the hypothesis that metacognition may be able to index differences between multisensory illusions and congruent multisensory information, but (in most circumstances) may be unable to index differences in the underlying causal structure which produces the sensory signals.

Keywords: Multisensory Integration, Metacognition, Sound-Induced Flash Illusion, Perceptual Metamer, Causal Metamer
\end{abstract}




\section{Metacognitive Awareness in the Sound-Induced Flash Illusion}

Physically distinct sensory stimuli that are perceptually indistinguishable are known as “perceptual metamers” (Wandell 1995; Loftus and Ruthruff 1994; Williams, Tweten, and Sekuler 1991). Examples of metamers can be found in many sensory modalities, including visual images with altered content in peripheral locations (Freeman and Simoncelli 2011), haptic stimuli which yield identical estimates of heaviness (Shockley, Carello, and Turvey 2004), and auditory stimuli that produce identical reports of spoken words (Feather et al. 2019). These examples demonstrate that perceptual sensitivity in any given domain is limited, and in specific circumstances, conditions can be found in which the brain's best estimate about the properties or identities of distinct sensory signals converges on the same solution.

Multisensory integration represents another example of perceptual metamers, as small misalignments in temporal or spatial properties of audiovisual stimuli can often result in the same perceptual report as judgments for fully congruent audiovisual stimuli (Deroy, Spence, and Noppeney 2016). For example, one well-known example of multisensory metamerism is the sound-induced flash illusion (Shams, Kamitani, and Shimojo 2000). In the "fission" version of this illusion, if observers are presented with two brief beeps and one visual flash, they often report seeing two visual flashes. In the "fusion" version of this illusion, if observers are presented with one beep and two visual flashes, they sometimes report seeing one visual flash (Andersen, Tiippana, \& Sams 2004; Mishra, Martinez, \& Hillyard 2008). Thus, participants' reports of the number of visual flashes in these illusory cases may be equivalent to reports in conditions with congruent audiovisual stimulation, where the number of flashes and beeps are the same. 
These conditions of distinct-stimulation-but-identical-report in the sound-induced flash illusion raise two interesting questions about perceptual metacognition. First, do observers display different estimates of metacognitive certainty in their judgments about the numbers of flashes across conditions? Previous research supports the hypothesis that phenomenological distinctions can be made between genuine flashes and illusory flashes (van Erp, Philippi, and Werkhoven 2013). Therefore, even if perceptual reports about the number of flashes are the same across conditions, it seems possible that metacognitive systems may be able to index differences by producing different levels of confidence. Second, can observers distinguish between conflicting and non-conflicting multisensory events, even when the perceptual report is the same? Models which assume that observers are performing a process akin to Bayesian causal inference have been effective in accounting for data in the sound-induced flash illusion (Shams, Ma, and Beierholm 2005; Körding et al. 2007). Thus, it remains an open question whether observers can metacognitively index the causal structure which gives rise to the sensory stimuli (i.e., whether the stimuli were produced by audiovisually congruent signals, or audiovisually incongruent signals) (Deroy, Spence, and Noppeney 2016).

In this investigation, we explored these two questions using the following version of the sound-induced flash illusion. On each trial, observers were presented with 1-4 flashes and 1-4 beeps, and were asked to judge three things: (1) the number of flashes that were presented, (2) their confidence in their judgment about the number of flashes, (3) their confidence in whether the number of stimuli presented in each modality were the same, or different. For our main analyses, we selected subsets of trials across conditions yielding perceptual metamers; specifically, we selected trials across pairs of conditions where the flash response was the same ("metamer trials"). To anticipate, results showed that for metamer trials with low numbers of 
stimuli in both the "fission" and "fusion" illusions, participants were significantly more confident in their response about the number of flashes when audiovisual stimuli were congruent than when they were incongruent. For metamer trials with larger numbers of stimuli, confidence was similar across conditions. Interestingly, for judgments about whether the number of beeps and flashes were the same or different on each trial, confidence did not significantly differ across any condition pairs except for one. These results provide preliminary evidence that while some perceptual metamers may be causal metamers in most circumstances, metacognition may still be able to index some differences between metameric stimuli that yield the same perceptual reports.

\section{Materials and Methods}

\section{Participants}

Twenty-two undergraduate students at the University of Florida (11 women, 11 men, mean age $=18.9$ years, $\mathrm{SD}=0.97)$ volunteered to participate to earn course credit. Participants began the experimental session by completing an informed consent procedure. All experimental procedures were conducted in accordance with the Declaration of Helsinki.

\section{Apparatus and Stimuli}

The experiment was controlled by a 2017 15-Inch Macbook Pro running MATLAB 2019a. Auditory stimuli were presented via Creative Pebble Desktop Speakers and consisted of sine-wave sounds, which we refer to as "beeps." Visual stimuli were presented on a Gateway VX900 CRT monitor set to a refresh rate of $85 \mathrm{~Hz}$, and consisted of white discs drawn on a black background for approximately one frame. These discs were drawn approximately one degree in size and presented four degrees below the fixation cross. The fixation cross consisted of a white 
cross in the middle of a black screen. Subjects were seated with their head in a chinrest located $50 \mathrm{~cm}$ away from the monitor.

The timing of the stimuli was as follows: for unisensory auditory stimuli with two or more beeps, each beep was separated by $60 \mathrm{msec}$; for unisensory visual stimuli with two or more flashes, each flash was separated by $60 \mathrm{msec}$. For multisensory stimuli, the timing of the visual and auditory stimulus trains for all conditions were centered with respect to one another, such that if the number of stimuli were the same, the timing was of each beep and flash was simultaneous, and if the number of stimuli differed, the midpoint of each stimulus train was aligned. Timing was verified with a Tektronix 2211 Digital Storage Oscilloscope using a photo diode and auditory coaxial cable plugged into the headphone jack.

\section{Procedure}

The trials were structured so that each began with a white fixation cross in the middle of a black screen for $1000 \mathrm{~ms}$, followed by the presentation of stimuli (see Figure 1). The white disc was centered on the screen approximately four degrees below fixation. "Unisensory" trials were composed of either only flashes or only beeps. "Bisensory" trials included either conflicting or non-conflicting numbers of both flashes and beeps. Stimulus presentation lasted for anywhere between $60 \mathrm{~ms}-240 \mathrm{~ms}$, depending on the combination of stimuli for each trial. Immediately following the presentation of stimuli, participants were asked to answer three questions on the computer keyboard. First, they reported the number of flashes they perceived. Next, they were asked to report their level of confidence in the number of flashes they reported on a scale from 1 to 4 , with $1=$ extremely confident and $4=$ not at all confident. Lastly (and only in "bisensory" trials), participants reported how confident they were that the number of beeps and the number of 
flashes were either the same or different, on a scale from 1 to 8 , with $1=$ "Confident Same," 4 and 5 = "Not Confident" and 8 labeled as "Confident Different."

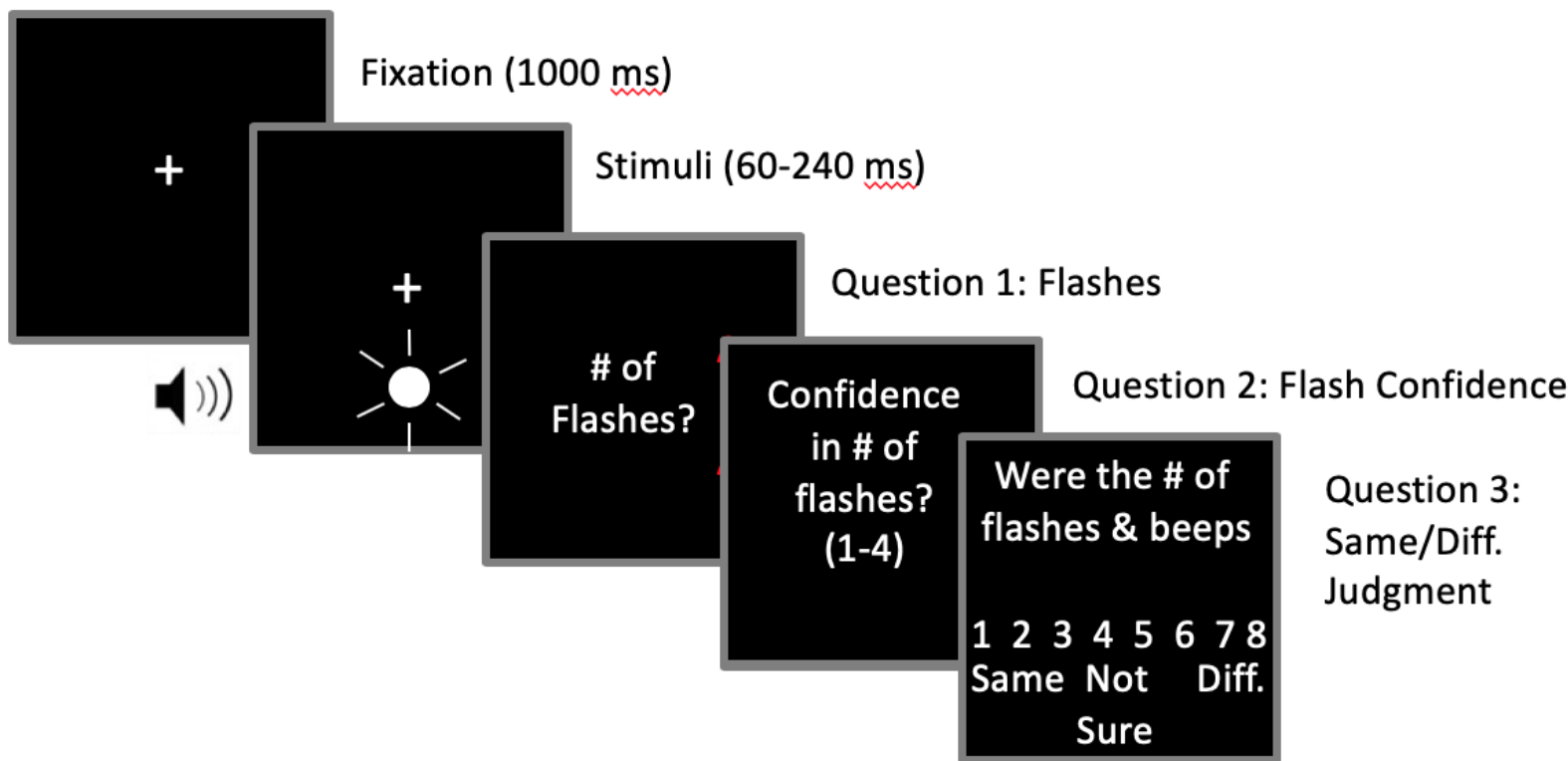

Figure 1. The experimental paradigm. On each trial, subjects began by fixating on a white cross for $1000 \mathrm{~ms}$. Next, stimuli were presented. Depending on the trial, this presentation could involve unisensory presentation (1-4 flashes or 1-4 beeps) or bisensory presentation (some combination of 1-4 flashes and beeps). Following the stimuli, participants were asked to report the number of flashes, their confidence in the judgment about the number of flashes on a scale from 1-4 ( 1 = extremely confidence, $4=$ not at all confident $)$, and their confidence in whether the number of flashes and beeps was the same on a scale from 1-8 (1 = "Confident Same," 4/5= "Not Confident," 8 = "Confident Different").

The general design of the paradigm was as follows: first, participants completed two short practice blocks of "unisensory" trials, where they were presented with either only flashes or only beeps. On each trial, participants would report the number of stimuli they perceived (from 1-4), and rate their confidence in their judgment on the scale from 1 to 4 . After completing 16 practice trials ( 8 visual-only trials, 8 auditory-only trials), participants began the real unisensory experiment blocks. Visual-only and auditory-only trials were pseudorandomly interleaved; each stimulus condition (1-4 flashes or 1-4 beeps) were presented 10 times for a total of 80 trials. As 
in the practice session, participants reported the number of stimuli and rated their confidence from 1-4 in their response. A self-guided break period was offered after 40 trials.

After completing the unisensory block, participants began a bisensory practice block with 16 trials. On any given trial, some combination of 1-4 flashes and 1-4 beeps was presented, and participants were asked to report three things: (1) the number of visual flashes, (2) how confident they were in that answer, on the scale from 1-4, (3) to rate their confidence in whether the number of beeps and flashes was the same on the scale from 1-8. Following the practice block, participants began the bisensory experiment block with 160 randomly interleaved bisensory trials, and after each trial, they were asked to answer the same three questions that were used in the practice block. Participants were allowed to take breaks every 40 trials. In total, the experiment took approximately 45 minutes to complete.

\section{Results}

For our first analysis, we selected all of the trials producing a "fission" sound-induced flash illusion and all of the trials from the congruent audiovisual conditions that correctly yielded the same perceptual report. This produced three pairs of conditions: "reported two flash" conditions (1-flash/2-beep and 2-flash/2-beep), "reported three flash” conditions (2-flash/3-beep and 3-flash/3-beep), and "reported four flash" conditions (3-flash/4-beep and 4-flash/4-beep). Next, we computed the average confidence in the flash response for these conditions for each subject, and then averaged across all subjects to evaluate differences between the conditions. 

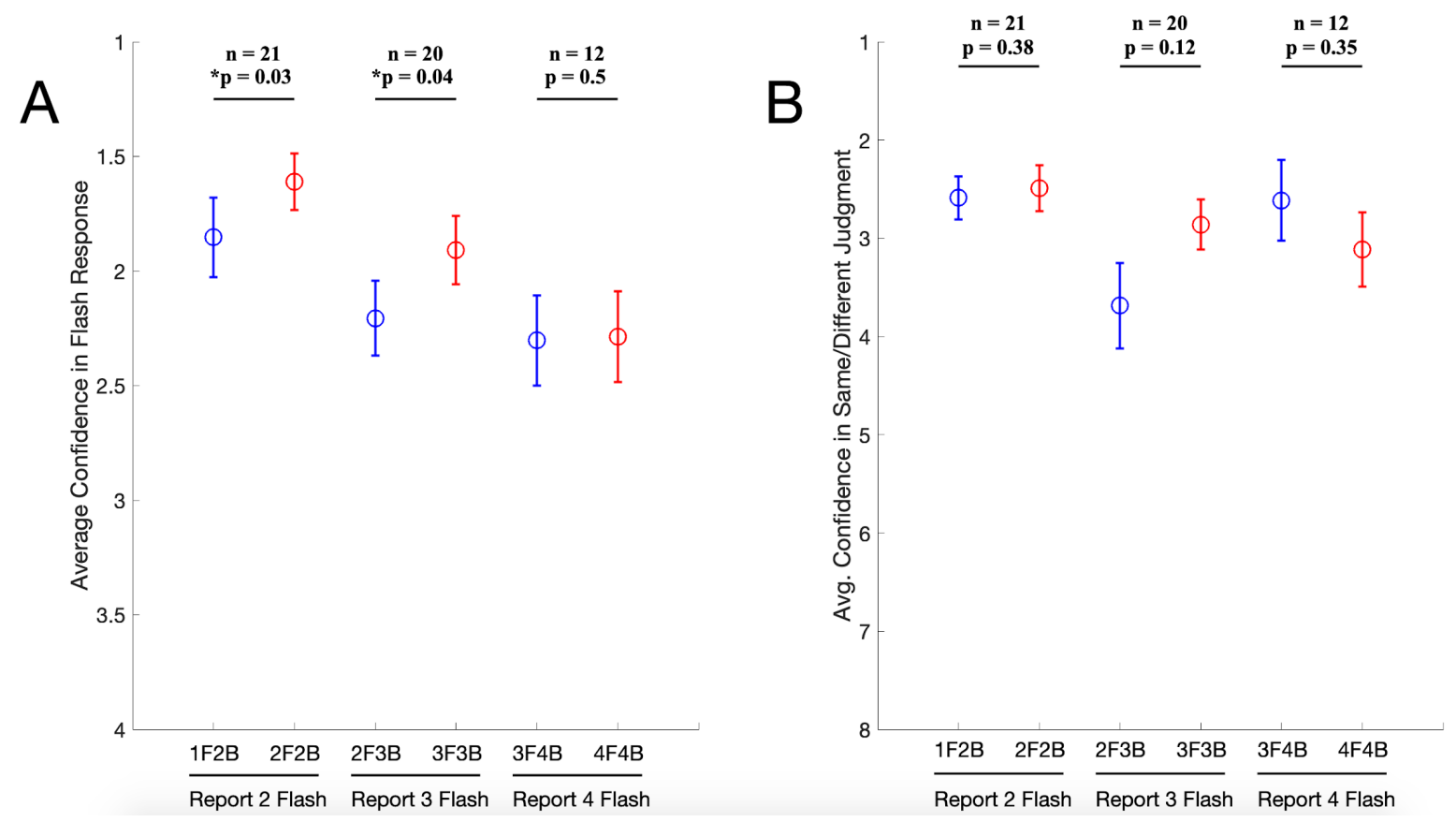

Figure 2. Results for "fission" trials \& audiovisually congruent trials with correct report. (A) Confidence in judgments about the number of flashes $(1=$ high confidence, $4=$ low confidence $)$. Conditions at the bottom are grouped by the identity of the metamer (reporting 2 flashes/ 3 flashes/4 flashes). For each participant, we first computed the average confidence in the judgment of the number of flashes for each condition, and then averaged across all subjects. Error bars represent SEM across subjects. (B) Confidence in whether the number of flashes and beeps were the same $(1=$ "Confident same" 4 and $5=$ "Not Confident" and 8 labeled as "Confident Different.").

As shown in Figure 2A, in the metamer trials where subjects reported 2 flashes (1flash/2-beep condition \& 2-flash/2-beep condition), subjects were more confident in their flash responses for the congruent stimuli than incongruent stimuli $(\mathrm{t}(20)=2.31, \mathrm{p}=0.03$, Cohen's $d=$ 0.51). The same general trend was evident for confidence responses in trials where subjects reported 3-flashes (2-flash/3-beep condition \& 3-flash/3-beep condition) $(\mathrm{t}(19)=2.23, \mathrm{p}=0.04$, Cohen's $d=0.50$ ). While these findings do not survive Bonferroni correction for multiple t-tests, the moderate effect sizes provide preliminary evidence that confidence may differ across conditions. Confidence for flash responses was quite similar in the metamer trials where subjects reported 4 flashes $(\mathrm{t}(11)=-0.70, \mathrm{p}=0.50$, Cohen's $d=-0.20)$. As shown in Figure $2 \mathrm{~B}$, 
for the same/different judgments, no differences in confidence were found in metamer trials across condition pairs (1-flash/2-beep condition \& 2-flash/2-beep condition: $\mathrm{t}(20)=0.91, \mathrm{p}=$ 0.38, Cohen's $d=0.20 ; 2$-flash/3-beep condition \& 3-flash/3-beep condition: $\mathrm{t}(19)=1.63, \mathrm{p}=$ 0.12, Cohen's $d=0.37 ; 3$-flash/4-beep condition \& 4-flash/4-beep condition: $\mathrm{t}(11)=-0.98, \mathrm{p}=$ 0.35, Cohen's $d=-0.28$ ). Taken together, these findings support two claims: (1) for a given perceptual report, confidence in a specific unimodal percept (i.e., the number of flashes) can differ across metamer conditions; (2) for a given perceptual report, confidence in whether the number of flashes and beeps are the same is similar across metameric conditions.

It should be noted that, consistent with previous findings, not all of our participants perceived each conflicting pair of multisensory events as a perceptual metamer (Stevenson, Zemtsov, and Wallace 2012; Odegaard and Shams 2016). Though we ran 22 participants in total, the number of individual data points analyzed for each condition was often less than that, as not all subjects experienced the illusion in each fission condition. For the 1-flash/2-beep conditions, we had 21 participants with data that exhibited a fission response on at least one trial; for 2flash/3-beep conditions, 20 participants; for 3-flash/4-beep conditions, 12 participants. Additionally, for 4-flash/4-beep non-conflicting multisensory pairs, only 16 of 22 participants reported the correct number of flashes in at least one trial, demonstrating the difficulty of subjects to parse a perception involving 4 flashes.

For our second analysis, we selected all of the trials producing a "fusion" sound-induced flash illusion and all of the trials from the congruent audiovisual conditions that correctly yielded the same perceptual report. This produced three pairs of conditions: "reported one flash" conditions (2-flash/1-beep and 1-flash/1-beep), "reported two flash" conditions (3-flash/2-beep and 2-flash/2-beep), and "reported three flash" conditions (4-flash/3-beep and 3-flash/3-beep). 
Next, we computed the average confidence in the flash response for these conditions for each subject, and then averaged across all subjects to evaluate differences between the conditions.

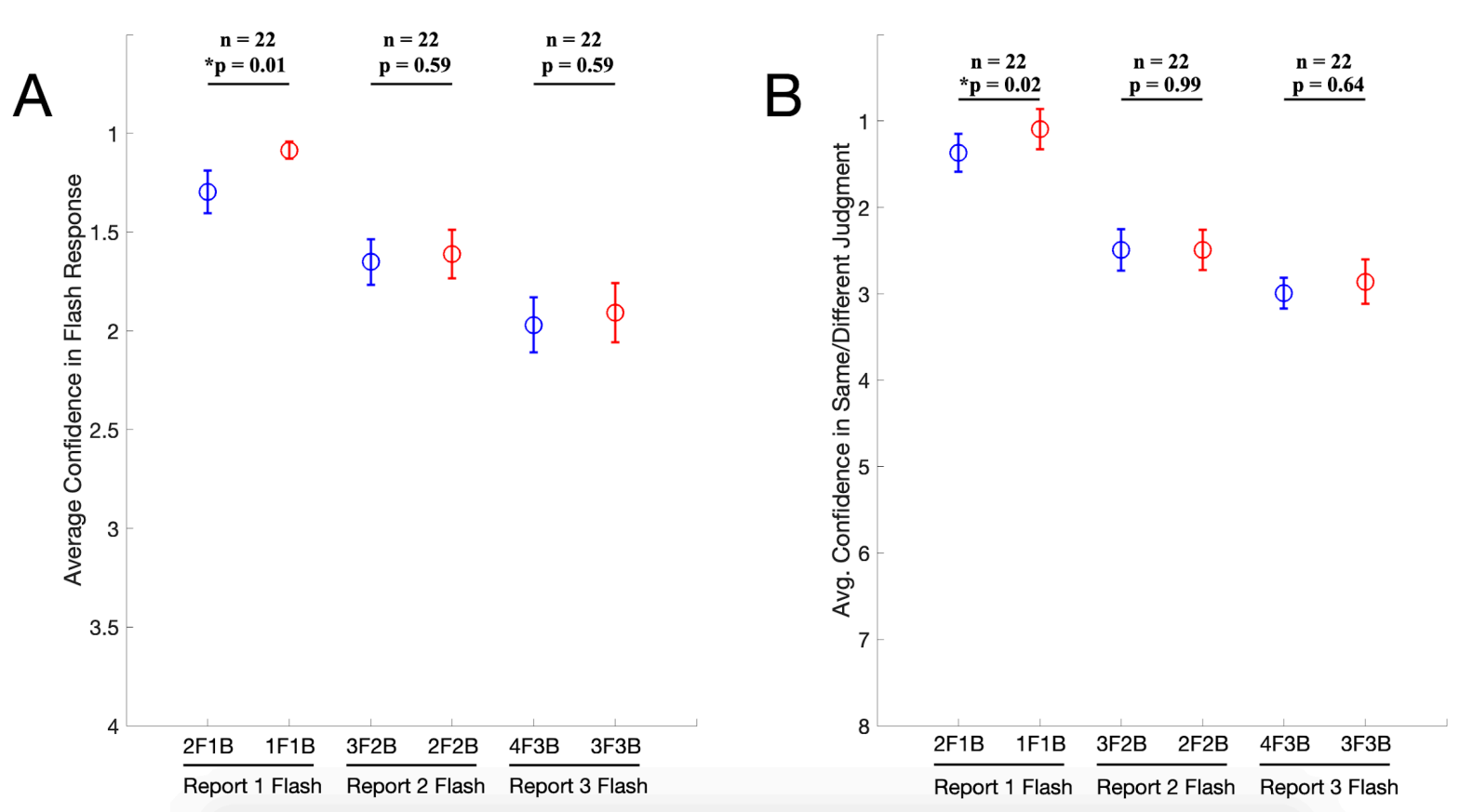

Figure 3. Results for "fusion" trials \& audiovisually congruent trials with correct report. (A) Confidence in judgments about the number of flashes $(1=$ high confidence, $4=$ low confidence $)$. Conditions at the bottom are grouped by the identity of the metamer (reporting 1 flash/2 flashes/3 flashes). For each participant, we first computed the average confidence in the judgment of the number of flashes for each condition, and then averaged across all subjects. Error bars represent SEM across subjects. (B) Confidence in whether the number of flashes and beeps were the same $(1=$ "Confident same" 4 and $5=$ "Not Confident" and 8 labeled as "Confident Different.").

As shown in Figure 3A, in the metamer trials where subjects reported 1 flash (2-flash/1beep condition \& 1-flash/1-beep condition), subjects were more confident in their flash responses for the congruent stimuli than incongruent stimuli $(\mathrm{t}(21)=2.8, \mathrm{p}=0.01$, Cohen's $d=$ 0.60). While this finding does not survive Bonferroni correction for multiple t-tests, the moderate effect size provides preliminary evidence that confidence may differ across conditions in this simple form of the fusion illusion. For the other metameric conditions, confidence was similar between pairs. This included conditions where two flashes were reported $(\mathrm{t}(21)=0.55, \mathrm{p}$ 
$=0.59$, Cohen's $d=0.12)$ and conditions where three flashes were reported $(\mathrm{t}(21)=0.55, \mathrm{p}=$ 0.59, Cohen's $d=0.12$ ). As shown in Figure 3B, for the same/different judgments, a trend for a difference in the same/different judgment was found in metamer trials where one flash was reported (2-flash/1-beep condition \& 1-flash/1-beep condition: $\mathrm{t}(21)=2.49, \mathrm{p}=0.02$, Cohen's $d$ $=0.53$ ). In the other conditions, same/different judgments were quite similar (3-flash/2-beep condition \& 2-flash/2-beep condition: $\mathrm{t}(21)=0.01, \mathrm{p}=0.99 ; 4$-flash/3-beep condition \& 3flash/3-beep condition: $\mathrm{t}(21)=0.47, \mathrm{p}=0.64$, Cohen's $d=0.20)$. Taken together, these findings indicate that for perceptual reports of only a single flash, confidence in a specific unimodal percept (i.e., the number of flashes) can differ across metamer conditions, and the same is true in this condition for judgments of whether the numbers of stimuli were the same or different. For larger numbers of stimuli in the fusion illusion, confidence in either type of judgment is similar across conditions. Finally, we note that unlike the fission conditions, each one of our participants appeared to experience at least one fusion illusion for each fusion condition presented. Because of this, we were able to maintain a sample size of $n=22$ for each fusion analysis.

\section{Discussion}

When we experience multisensory illusions, do we possess metacognitive knowledge about the nature of the illusion? Many behavioral studies have documented scenarios in which incongruent information across visual and auditory modalities can be combined to form an integrated percept. These cases sometimes yield the same perceptual reports about the identity of the stimulus as scenarios where the visual and auditory information is congruent. While examples of multisensory perceptual metamers (based on behavior) are well-documented in the domains of spatial (Welch 1974), temporal (Shams, Kamitani, and Shimojo 2000), and speech 
perception (McGurk and MacDonald 1976), little is known about how confidence judgments in a given percept compare across conditions congruent and incongruent audiovisual information, and whether confidence judgments can index differences in the underlying causal structure which produced the stimuli (Körding et al. 2007).

In this short report, we investigated confidence in the sound-induced flash illusion. On each trial, in addition to reporting the number of perceived flashes, observers had to report their confidence in the flash report, as well as their confidence in whether the perceived number of flashes were the same or different. For conditions with low numbers of stimuli, our results revealed trends that confidence in the perceived number of flashes may be higher for congruent audiovisual signals compared to incongruent audiovisual signals. For higher numbers of stimuli, confidence across metamer-pair conditions were quite comparable. Regarding the same/different judgment, we showed that confidence was slightly higher in the congruent 1-flash/1-beep condition when a single flash was reported compared to the 2-flash/1-beep condition, but for the rest of the metamer pairs, confidence in the same/different judgment was extremely similar.

How do these results compare to other studies in the literature? Relatively few studies have investigated confidence judgments in the sound-induced flash illusion. One previous investigation with a small sample size $(\mathrm{N}=9)$ used large numbers of visual and auditory stimuli (2-6) in different conditions and failed to find differences in confidence across conditions (Abadi and Murphy 2014). Importantly, in our investigation, we find that the differences in confidence are most evident in conditions with smaller numbers of stimuli, and that it is difficult for observers to perceive high numbers of flashes. This may account for differences in the previous investigation and our study here. Another important study found that if observers are given a third response option in the simple version of the beep-flash illusion task using 1-2 flashes, (i.e., 
being allowed to report that their percept was 'different from that of either 1 or 2 flashes'), on nearly half of the illusion trials, observers choose this third option (van Erp, Philippi, and Werkhoven 2013). This opens up an intriguing hypothesis: perhaps claims of multisensory metamerism are too strong, and that illusory percepts can often be distinguished from congruent audiovisual percepts. Additional experiments that go beyond 2-AFC paradigms in this task are needed to further parse these effects.

These results motivate future work in three main ways. First, this pilot investigation represents a promising paradigm that can be replicated and extended in other laboratories. For the sound-induced flash illusion, the primary conditions of interest may be standard versions of the illusion with lower numbers of stimuli, but future research can probe other domains of multisensory integration, including the ventriloquist illusion (Welch and Warren 1980) and McGurk speech tasks (McGurk and MacDonald 1976). Second, additional work is needed to determine how question type \& question order may influence metacognitive responses. For example, if the same/different judgment question is asked by itself (without the preceding judgments about the number of flashes or confidence in that report), would the results be the same? Or if the confidence in the flash report is asked as the last question, would the results still hold? More work is needed to answer these questions. Third, based on these results, one intriguing hypothesis is that prestimulus oscillations may drive not only confidence in the illusory percept, but also the percept of the illusory flash itself. For instance, it has been shown that oscillations can predict susceptibility to the sound-induced flash illusion on a trial-by-trial basis (Kaiser et al. 2019). Prestimulus alpha band power has also been shown to be linked to confidence judgments, but not perceptual accuracy (Samaha, Iemi, and Postle 2017). Thus, research which combines this behavioral paradigm with EEG to measure prestimulus oscillations 
could determine which prestimulus components drive behavioral reports and metacognitive biases produced by these conditions.

The study of how multisensory integration and metacognition relate to one another is only in its infancy. Recent contributions to the scientific literature have outlined promising paradigms and modeling approaches to make progress in this area (Deroy, Spence, and Noppeney 2016). Here, we provide preliminary evidence that metacognitive differences may be present across pairs of audiovisual metamers, and outline future work to further address this question. We think that preregistered studies with power analyses based on these results will prove useful in seeing whether our findings can be replicated, and look forward to additional work probing the neural basis of these effects. 


\section{References}

Anderson, Tobias S., Kaisa Tiippana, and Mikko Sams. 2004. "Factors Influencing Audiovisual Fission and Fusion Illusions." Cognitive Brain Research 21 (3): 301-308.

Abadi, Richard V., and Jonathan S. Murphy. 2014. "Phenomenology of the Sound-Induced Flash Illusion." Experimental Brain Research. Experimentelle Hirnforschung. Experimentation Cerebrale 232 (7): 2207-20.

Deroy, Ophelia, Charles Spence, and Uta Noppeney. 2016. "Metacognition in Multisensory Perception." Trends in Cognitive Sciences 20 (10): 736-47.

Erp, Jan B. F. van, Tom G. Philippi, and Peter Werkhoven. 2013. "Observers Can Reliably Identify Illusory Flashes in the Illusory Flash Paradigm.” Experimental Brain Research. Experimentelle Hirnforschung. Experimentation Cerebrale 226 (1): 73-79.

Feather, Jenelle, Alex Durango, Ray Gonzalez, and Josh McDermott. 2019. "Metamers of Neural Networks Reveal Divergence from Human Perceptual Systems." In Advances in Neural Information Processing Systems 32, edited by H. Wallach, H. Larochelle, A. Beygelzimer, F. dltextquotesingle Alché-Buc, E. Fox, and R. Garnett, 10078-89. Curran Associates, Inc.

Freeman, Jeremy, and Eero P. Simoncelli. 2011. "Metamers of the Ventral Stream." Nature Neuroscience 14 (9): 1195-1201.

Kaiser, Mathis, Daniel Senkowski, Niko A. Busch, Johanna Balz, and Julian Keil. 2019. "Single Trial Prestimulus Oscillations Predict Perception of the Sound-Induced Flash Illusion." Scientific Reports 9 (1): 5983.

Körding, Konrad P., Ulrik Beierholm, Wei Ji Ma, Steven Quartz, Joshua B. Tenenbaum, and Ladan Shams. 2007. "Causal Inference in Multisensory Perception." PloS One 2 (9): e943.

Loftus, Geoffrey R., and Eric Ruthruff. 1994. "A Theory of Visual Information Acquisition and Visual Memory with Special Application to Intensity-Duration Trade-Offs." Journal of Experimental Psychology. Human Perception and Performance 20 (1): 33-49.

McGurk, H., and J. MacDonald. 1976. "Hearing Lips and Seeing Voices.” Nature 264 (5588): 746-48. Mishra, Jyoti, Antigona Martinez, and Steven A. Hillyard. 2008. "Cortical Processes Underlying Sound-Induced Flash Fusion.” Brain Research 1242: 102-115.

Odegaard, Brian, and Ladan Shams. 2016. "The Brain's Tendency to Bind Audiovisual Signals Is Stable but Not General.” Psychological Science 27 (4): 583-91.

Samaha, Jason, Luca Iemi, and Bradley R. Postle. 2017. "Prestimulus Alpha-Band Power Biases Visual Discrimination Confidence, but Not Accuracy." Consciousness and Cognition 54 (September): $47-$ 55.

Shams, Ladan, Wei Ji Ma, and Ulrik Beierholm. 2005. "Sound-Induced Flash Illusion as an Optimal Percept.” Neuroreport 16 (17): 1923-27.

Shams, L., Y. Kamitani, and S. Shimojo. 2000. "Illusions. What You See Is What You Hear." Nature 408 (6814): 788.

Shockley, Kevin, Claudia Carello, and M. T. Turvey. 2004. "Metamers in the Haptic Perception of Heaviness and Moveableness." Perception \& Psychophysics 66 (5): 731-42.

Stevenson, Ryan A., Raquel K. Zemtsov, and Mark T. Wallace. 2012. "Individual Differences in the Multisensory Temporal Binding Window Predict Susceptibility to Audiovisual Illusions." Journal of Experimental Psychology. Human Perception and Performance 38 (6): 1517-29.

Wandell, Brian A. 1995. Foundations of Vision. Sinauer Associates.

Welch, R. B. 1974. "Research on Adaptation to Rearranged Vision: 1966-1974.” Perception 3 (4): $367-$ 92.

Welch, R. B., and D. H. Warren. 1980. "Immediate Perceptual Response to Intersensory Discrepancy." Psychological Bulletin 88 (3): 638-67.

Williams, D., S. Tweten, and R. Sekuler. 1991. "Using Metamers to Explore Motion Perception." Vision Research 31 (2): 275-86. 